\title{
Téoros
}

Revue de recherche en tourisme

\section{Le tourisme médical, une nouvelle façon de voyager}

\section{Loïck Menvielle et William Menvielle}

Volume 29, numéro 1, 2010

URI : https://id.erudit.org/iderudit/1024761ar

DOI : https://doi.org/10.7202/1024761ar

Aller au sommaire du numéro

Éditeur(s)

Université du Québec à Montréal

\section{ISSN}

0712-8657 (imprimé)

1923-2705 (numérique)

Découvrir la revue

Citer cet article

Menvielle, L. \& Menvielle, W. (2010). Le tourisme médical, une nouvelle façon de voyager. Téoros, 29(1), 109-119. https://doi.org/10.7202/1024761ar

\section{Résumé de l'article}

Même si ses racines remontent à l'antiquité, la dénomination « tourisme médical » est fort récente et désigne les patients désireux de se faire soigner à l'étranger à des prix abordables, profitant d'une expertise médicale et d'un décor différent de celui de leur quotidien pour leur convalescence. Le but de cet article est de présenter ce concept et d'analyser les facteurs qui le stimulent, de même que les activités de marketing-mix mises en place par les cliniques pour capter ces patients d'un " nouveau genre " et minimiser le risque perçu par ces derniers. Un regard sur l'avenir est aussi proposé en matière de couple produit-marché afin d’envisager les perspectives à développer. 


\title{
Le tourisme médical, une nouvelle façon de voyager
}

\author{
Loïck MENVIELLE \\ Maître de conférences \\ Edhec Business School \\ loick.menvielle@edhec.edu \\ William MENVIELLE \\ Professeur \\ Université du Québec à Trois-Rivières \\ William.Menvielle@uqtr.ca
}

\begin{abstract}
RÉSUMÉ: Même si ses racines remontent à l'antiquité, la dénomination «tourisme médical » est fort récente et désigne les patients désireux de se faire soigner à l'étranger à des prix abordables, profitant d'une expertise médicale et d'un décor différent de celui de leur quotidien pour leur convalescence. Le but de cet article est de présenter ce concept et d'analyser les facteurs qui le stimulent, de même que les activités de marketing-mix mises en place par les cliniques pour capter ces patients d'un «nouveau genre» et minimiser le risque perçu par ces derniers. Un regard sur l'avenir est aussi proposé en matière de couple produit-marché afin d'envisager les perspectives à développer.
\end{abstract}

Mots-clés: Médecine, santé, tourisme, risque, classification.

Après un début des années 2000 en reprise, suite aux événements du 11 septembre 2001, le nombre de touristes a cru de $2 \%$, entre 2007 et 2008, passant de 908 à 924 millions (Organisation mondiale du tourisme, 2010). Cependant, à l'instar de nombreux autres secteurs d'activités, l'activité touristique a connu un repli en enregistrant une baisse des voyageurs pour 2009 , de l'ordre de $4 \%$ pour s'établir à 880 millions. Plusieurs facteurs en sont la cause, comme la crise économique et la pandémie de grippe H1N1. Les résultats des arrivées internationales de touristes pour 2008 montrent que cinq pays arrivent en tête des destinations touristiques, à savoir la France (78 millions de touristes) qui maintient sa place de destination phare au niveau mondial, talonnée par les ÉtatsUnis (58), l'Espagne (57), la Chine (53) et l'Italie (42) venant se greffer à ce peloton de tête des piliers du tourisme. Bien que les prévisions générales ainsi que les projections émises par l'OMT pour 2010 soient favorables au secteur touristique dans son ensemble, après une année 2009 difficile, il est à noter que la concurrence est grandissante et que de nombreux pays rivalisent d'ingéniosité et de finesse marketing pour attirer cette multitude de visiteurs et leur argent. On constate alors l'émergence de nouvelles tendances pour attirer des touristes en quête d'exotisme d'un nouveau genre.

Le concept du «bronzer idiot» semble être passé de mode, et la filière touristique a connu quelques changements parfois radicaux, voire surprenants, alors même que le secteur s'inscrit dans une démarche que nous pouvons qualifier de traditionnelle comparativement à d'autres domaines. Selon Lozato-Giotart et Balfet (2007 : 104), «l'évolution des pratiques de loisirs et de tourisme reste étroitement liée à l'évolution socioculturelle des pays émetteurs", donnant ainsi le ton à de nouvelles pratiques. Certains parlent ainsi d'un «post-tourisme» qui n'est plus centré sur le simple fait de délivrer des voyages aux consommateurs, mais sur celui d'analyser les différentes interactions qui puissent exister, en se confrontant notamment aux réalités sociales, environnementales et humaines dans les zones d'accueil des touristes. Le recours aux modèles traditionnels du comportement du consommateur pour analyser ces tendances est un fait; travailler le marché dans sa globalité sans mettre en exergue des motivations et des attentes particulières pour les individus serait une pure aberration. Ainsi pouvons-nous, à la lumière de ces modèles, déceler chez certains consommateurs une farouche volonté de découvrir de nouvelles cultures, de s'imprégner d'un passé plus ou moins ancien, témoin de leurs origines : le tourisme culturel est né, et avec lui des canaux particulier de distribution, uniquement spécialisés sur ce segment à l'instar des agences Donatello (www.donatello.fr) en France. On note également l'avènement d'un tourisme écologique autour de la notion de développement durable. 
Parallèlement à ce constat, apparaît depuis quelques années l'existence d'un tourisme industriel, pour les curieux désireux de connaître ou de comprendre les rouages des entreprises développant les produits et services qu'ils aiment et consomment comme chez Bombardier ou Vachon au Québec. Mais au-delà de ces tendances, qui ont leur intérêt, nous prenons le temps de nous attarder sur un des phénomènes récents et non des moindres qu'est le tourisme médical.

Pour ce faire, nous commençons par présenter un historique de ce concept. Dans un second temps, nous expliquons notre vision du tourisme médical moderne et ses différents enjeux. Partant des travaux de Mueller et Lanz Kaufmann (2001), nous proposons un nouveau cadre conceptuel relatif au tourisme médical. Notre point de vue concerne le touriste en tant que patient, son degré d'implication dans ce processus de sélection d'un service de santé. À cet effet, une nouvelle classification sera proposée afin de mieux apprécier tout l'intérêt de ce concept.

Dans une fin de partie, nous présentons les variables incitatives mises en place pour attirer ces touristes d'un nouveau genre et nous terminerons notre analyse par un panorama de quelques stratégies de diversification choisies par divers pays.

\section{Le tourisme médical, une tradition séculaire}

Pour de nombreux individus, le tourisme médical semble être un terme abscons, un néologisme douteux, sans réelle signification. Pourtant, le tourisme médical est indissociable de l'histoire des civilisations avec laquelle il partage de nombreux points en commun. Les Égyptiens avaient compris très tôt les vertus curatives des bains de mer pour soulager des douleurs ou des problèmes de santé. Mais c'est surtout sous l'égide des Grecs et des Romains que s'est développée cette tradition de l'hydrothérapie, l'exploitation des sources d'eau à des fins médicales et le recours à l'eau chaude comme remède miracle. Pour Smith et Kelly (2006), ce type de tourisme à la recherche du bien-être (aller à un endroit pour bénéficier des propriétés des eaux ou des qualités des boues thermales) est l'une des formes les plus anciennes de tourisme qui soit donnée de recenser.

Cette quête du bien-être et des possibilités de soigner des maladies, parfois considérées comme «incurables» pour l'époque, a incité certaines populations à émigrer vers l'Empire romain pour bénéficier de tels soins. La quintessence même de l'hydrothérapie, la thalassothérapie, ainsi que des formes plus «ordinaires» comme l'utilisation des sources d'eaux naturelles ou thermales, ont été largement exploitées jusqu'au Moyen Âge. L'obscurantisme, synonyme de cette époque, a contribué au déclin de cette exploitation du thermalisme et des bienfaits des eaux.

Ce n'est qu'au dix-huitième siècle que l'on a redécouvert les propriétés de l'eau faisant ainsi renaître le tourisme médical. En Europe, la ville de Bath au Royaume-Uni est l'illustration parfaite de cette réappropriation du thermalisme (Boyer, 1996). Les aristocraties anglaise et européenne avaient fait de ce lieu l'endroit de référence où il fallait tout aussi bien être pour se montrer que pour bénéficier de soins jugés performants pour l'époque. En France, c'est sous l'impulsion de Napoléon III que les thermes bénéficient d'un

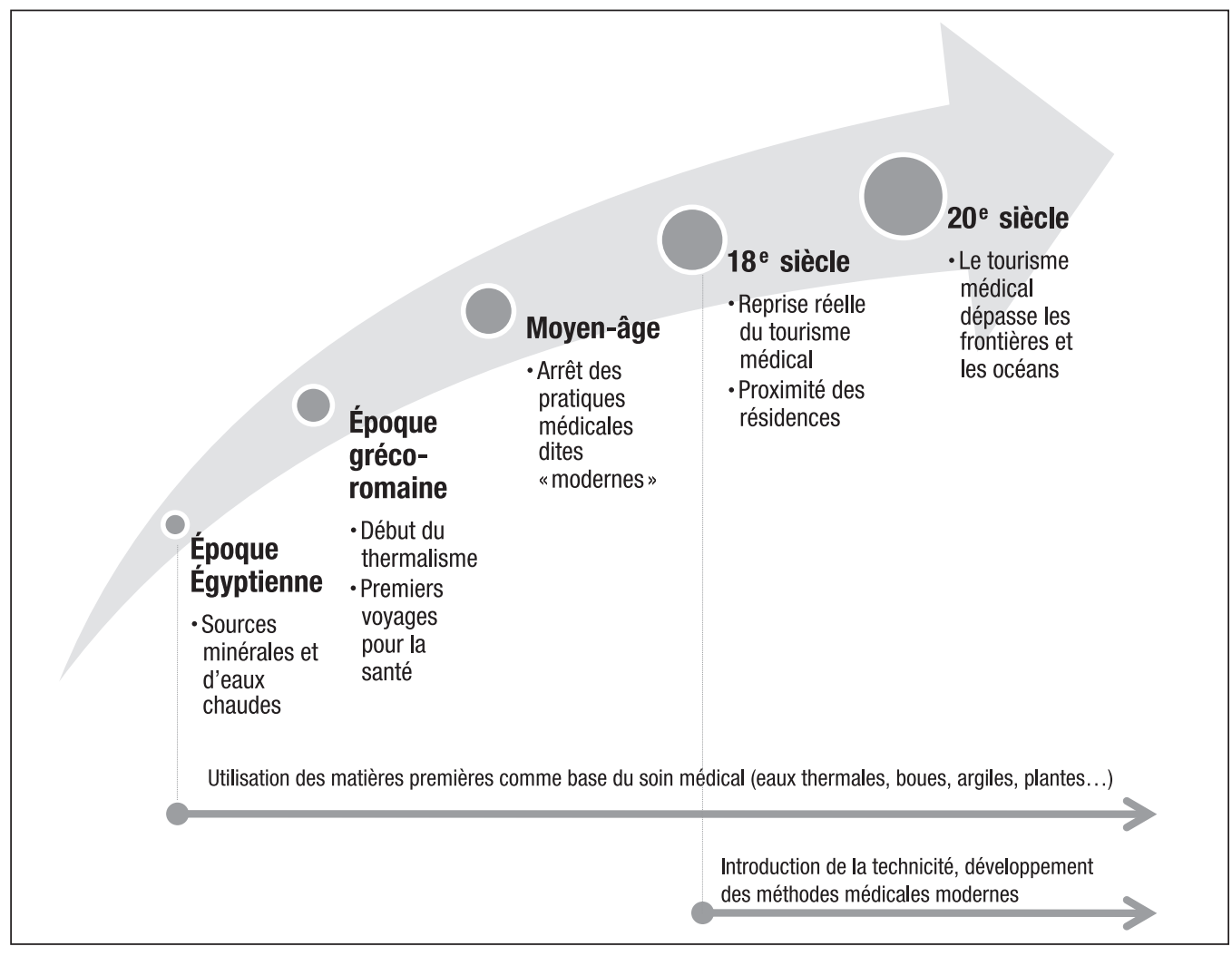

ILLUSTRATION 1 : Évolution du tourisme médical au cours des âges (source : Menvielle et Menvielle).

TÉOROS, vol. 29, n 1, p. 109-119, ( $) 2010$ 
nouvel engouement du public. Au début du vingtième siècle, la Côte d'Azur attire les têtes couronnées de ce monde, puis l'aristocratie de façon plus large, autour de ce que l'on peut qualifier d'esquisse du tourisme médical (Smith et Kelly, 2006; Proulx, 2005). Boyer (1996) parle même d'un véritable phénomène de tourisme médical moderne, cette région du sud-est de la France étant considérée comme propice par son climat pour se soigner et se rencontrer tout à la fois entre gens de ce monde.

L'illustration 1 évoque l'évolution du tourisme médical au cours des siècles, passant ainsi de la période égyptienne, constituant les soubassements du tourisme médical, à aujourd'hui. Avec le temps s'instaurent de nouvelles tendances autour de ce type de tourisme. Les changements les plus significatifs surviennent après la Seconde Guerre mondiale, à une époque où l'accélération des phénomènes sociaux, politiques, économiques et logistiques se traduisent par une augmentation de l'activité touristique, des loisirs et du divertissement (Gartner et Lime, 2000).

C'est ce développement technologique, allié au progrès social, et l'expansion économique qui permettront d'arriver à la démocratisation du tourisme et des loisirs. Désormais, le monde n'a plus réellement de frontières, plus de limites. L'essor et surtout la popularisation des moyens de transport (rail, route et air) ayant fortement contribué à cette démocratisation des voyages, ce n'est plus uniquement une élite qui a accès aux destinations éloignées, mais bientôt toute une classe moyenne, inscrite dans ce courant que certains envisageaient comme la société des loisirs (Gilbert, 1954), ce dont certains doutent et que d'autres cherchent encore.

Mours des temps modernes, le travail a pris le dessus au détriment de la société des loisirs. La performance et la compétitivité en sont devenues les maîtres mots, si bien que sous le poids de la fatigue ou des problèmes de santé qui en découlent parfois, une forme moderne de tourisme est née, apportant une réponse à ces fléaux. Le tourisme médical a pris naissance, qui connaît une forte expansion depuis une décennie.

Véritable mode passagère introduite par les gestionnaires marketing? Nouvelles lubies de consommateurs (Kalfon, 2009) ou réelle réponse destinée à pallier aux carences des systèmes en place dans certains pays? Il convient de s'interroger sur le sujet. Avant de cerner les enjeux qui émanent du tourisme médical, tout en expliquant les différences subtiles entre ses différentes formes, faisons un tour d'horizon des facteurs permettant de fournir un terreau fertile au développement du tourisme médical avant d'analyser les stratégies marketing à emprunter par les pays proposant ce genre d'activité.

\section{La version moderne du tourisme médical : un degré d'implication différent selon le risque perçu par le patient}

Les propos tenus au début de cet article nous permettent de comprendre que le tourisme médical - même si la dénomination est récente - est une forme relativement ancienne de tourisme, qui a connu un essor tardif. La méconnaissance de ce domaine vient du fait que les termes associés au tourisme médical relèvent du secteur de la santé. En ce sens, le domaine peut paraître hermétique, voire affolant pour de nombreux consommateurs. Qui plus est, la communauté scientifique s'est pour le moment peu intéressée à ce phénomène, laissant la place aux journalistes évoquant des destinations soleil, paradis terrestres pour se reposer... après s'être fait soigner. Il y a un réel manque d'investissement sur ce champ de recherche, notamment en ce qui concerne la production scientifique qui reste pour le moment très marginale sur le sujet et n'aborde pas réellement tous les enjeux et les concepts sous-jacents destinés à appréhender des questions fondamentales sur notre société et sur la marchandisation de la santé. L'objet de cet article est de centrer notre analyse sur les flux Nord-Sud reliés au tourisme médical qui constituent l'axe le plus important dans des déplacements d'individus. Bien évidemment, le tourisme médical au sens large englobe les échanges Sud-Sud qui connaissent une progression rapide à l'instar du développement de la Jordanie ou de la Tunisie qui aujourd'hui privilégient un nouvel axe de commercialisation de ces voyages à forfait médicaux en direction des pays arabes (Lautier, 2007). Les relations Sud-Nord et Nord-Nord constituent également des axes du tourisme médical mais dont l'importance est à reléguer au second plan (Menvielle et al., 2010).

Pour certains chercheurs comme Garcia-Altes (2005) le tourisme médical recouvre une pluralité de réalités, en raison des nombreuses pratiques induites par le secteur de la santé. Nous pouvons ainsi distinguer dans la dominante du tourisme de santé ou médical deux axes principaux : le tourisme médical de bien-être ou de confort et le tourisme médical vital. Même si la frontière entre ces deux termes est mince, il convient dans l'approche marketing et stratégique de signifier le distinguo. Pour certains, le tourisme de bien-être se définit selon des vertus curatives ou de soins chirurgicaux destinés à embellir la personne plutôt que de la soigner à l'instar de la chirurgie plastique, alors que le tourisme médical vital est avant tout de nature à répondre à de lourdes pathologies que peuvent avoir les patients. De la même façon, il nous apparaît indispensable de définir la notion de touriste-patient, un terme peu usité, mais qui reflète une certaine réalité de la situation à laquelle nous sommes confrontés.

Le touriste-patient, néologisme que nous avons créé, désigne un patient nécessitant une intervention chirurgicale, plus ou moins importante, et désireux de partir à l'étranger pour bénéficier de soins. Ainsi, nous évoquerons par exemple, le fait que des patients anglais ou américains partent en Inde pour bénéficier de certains soins. De cette définition est ainsi exclu le patient qui attend de se faire opérer sur le lieu même où il réside (Anglais en Angleterre, Américain aux États-Unis pour poursuivre avec notre exemple). Qui plus est, dans le cadre du tourisme médical, le touriste-patient utilise l'infrastructure touristique complète mise à sa disposition : voyage, hébergement, ou le recours à une agence de voyage spécialisée, autorisée (ou tolérée) seulement dans certains pays.

De nombreuses variables expliquent le comportement des patients pour choisir une destination éloignée afin de se faire soigner. Signalons ainsi les longues listes d'attentes opératoires (Bovier, 2008), le prix des chirurgies (Walker, 2006), des traitements parfois jugés peu encourageants par les patients, souhaitant tenter leur « ultime chance de survie 
à l'étranger» et le fait que certaines compagnies d'assurances n'assument pas la couverture complète des dépenses ou n'assurent pas l'intervention pratiquée par le chirurgien (Le Borgne, 2007).

Cependant, le terme de tourisme de bien-être est une appellation générique qu'il convient de distinguer. En reprenant les travaux de Mueller et Lanz Kaufmann (2001), et en analysant la réalité du marché, nous avons ainsi développé la classification suivante :

- Le tourisme médical de bien-être ou de confort : ces offres répondent à des besoins de confort des touristes-patients. Ainsi passons-nous des voyages à forfait de chirurgie esthétique aux soins préventifs tels que les spas, la thalassothérapie, les forfaits de relaxation (proximité de la nature) qui constituent quelques activités typiques du secteur, parfois associées au tourisme durable, notamment sur les axes marchands dernièrement cités. À cet effet, il est possible d'envisager d'éventuels liens commerciaux entre ces deux pratiques touristiques.

- Le tourisme médical vital qui peut se différencier en deux sous-ensembles :

-Le tourisme médical de réhabilitation : il s'agit ici de soins médicaux généraux destinés à pallier aux problèmes vécus par les individus. Nous citerons à titre d'exemples les traitements contre des dépendances de toutes formes (tabagisme, drogue, alcool). Mais il convient d'y adjoindre également les soins de dialyse pour les insuffisants rénaux. Aussi surprenant que cela puisse paraître, de nombreux centres en Tunisie ouvrent des complexes ${ }^{1}$ spécialement dédiés à ces individus et parfois même dans des zones reculées.
-Le tourisme médical opératoire : il nécessite une infrastructure médicale complète, associée à un personnel hautement qualifié (médecins, chirurgiens, anesthésistes...). C'est sans doute l'activité la plus connue, du moins la plus médiatisée auprès du grand public en raison de son vaste potentiel d'intervention (chirurgie dentaire, cardiaque, traitements anticancéreux). Elle correspond à des besoins particuliers des consommateurs qui ne peuvent être traités dans les meilleures conditions dans leurs pays d'origine (listes d'attente, coûts des opérations, par exemple).

Le premier concept de notre classification correspond au tourisme de bien-être, de confort, tandis que les deux autres émanent du tourisme chirurgical, répondant à des notions vitales et parfois même essentielles pour les individus. Nous pouvons ainsi établir une distinction entre ces différentes formes de tourisme par le biais de la nature des soins prodigués, mais aussi selon le degré de risque perçu par les consommateurs. L'illustration 2 ci-après constitue une synthèse de nos propos, en complétant les travaux de Mueller et Lanz Kaufmann (2001). Si nous avons repris les notions de bases de ces deux auteurs, nous avons ajouté les divers degrés d'implication des consommateurs (des patients) à l'égard de l'offre médicale, son degré de risque perçu et également son interaction avec l'infrastructure globale (le risque perçu peut se définir comme une incertitude associée aux conséquences négatives d'un choix. Plusieurs types de risques existent comme nous le présenterons dans un paragraphe ultérieur).

En conséquence, trois types de soins sont proposés au patient, que ce soit pour des raisons préventive, palliative ou curative. Pour chacun de ces concepts, nous avons introduit la notion de risque perçu qui peut varier d'un extrême

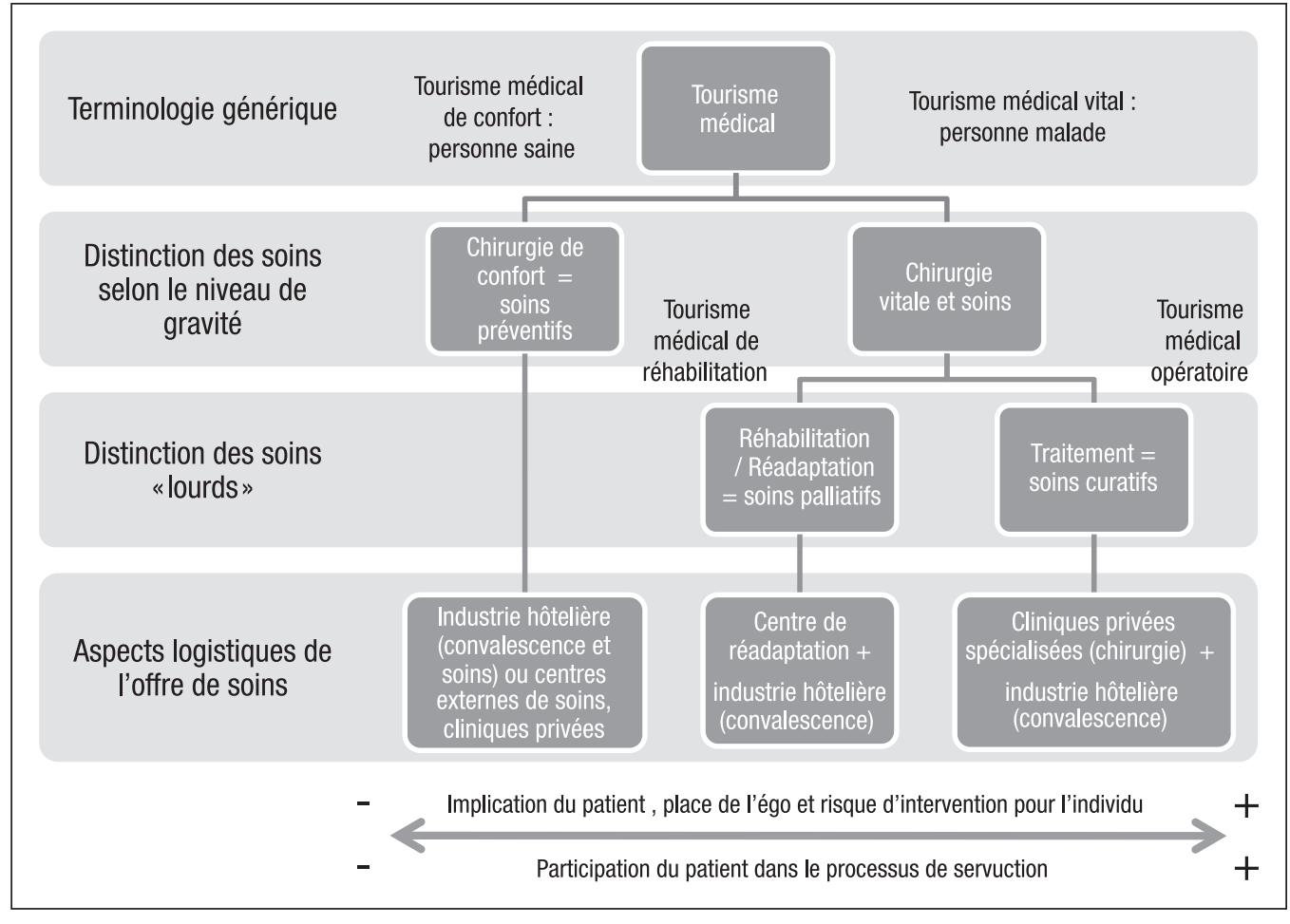

ILLUSTRATION 2 : La diversité des offres du tourisme médical (source : adapté de Mueller et Lanz Kaufmann (2001: 8) et complété par Menvielle et Menvielle, 2007 et 2010). 
à un autre. Ainsi un patient en recherche de soins de type hydrothérapie n'aura pas la même appréhension qu'une personne nécessitant un changement de valve cardiaque. De même, l'aggravation d'une maladie pourrait également baisser le niveau de risque perçu, faisant baisser globalement l'appréhension du touriste-patient. Toutefois, nous n'avons pas souhaité, à cette étape d'analyse, présenter l'ensemble de ces spécificités.

Contrairement à Mueller et Lanz Kaufmann (2001), il convient, d'évoquer les éléments touchant directement l'intégrité morale, psychique et physique des patients, ceci en guide d'adaptation à notre champ d'application. Cette analyse de base nous permet ainsi d'élaborer des stratégies marketing particulières utilisées par certains hôpitaux des pays pratiquant le tourisme médical.

Une des principales questions sous-jacentes concerne la façon de réduire le risque perçu auprès du consommateur afin d'engendrer une démarche favorable d'achat. Cette question est primordiale dans le marketing des services, caractérisé par son intangibilité et elle ressort comme l'une des préoccupations majeures de ces dernières années puisqu'on en retrouve des prémisses dans les travaux de Bauer (1960), Güseman (1981), Flipo (1984, 1988), Murray et Chlater (1990), Laroche et al. (2001, 2003) ou encore Mallet (2004). L'influence négative du risque perçu est encore plus importante dans le secteur médical où elle peut engendrer une forme de répulsion.

Suite aux commentaires que nous avons formulés sur l'illustration 2 précédente, nous comprenons aisément que le niveau d'implication de l'individu, aussi bien dans le cas de la chirurgie lourde que des soins ou des traitements de réadaptation, se situe à des stades identiques à première vue.

Ainsi, la perception du risque est proportionnelle au type de soin subi; il est plus important pour le patient dans le cas d'une chirurgie lourde (chirurgie cardiaque) que d'un soin de balnéothérapie. Cette analyse du risque reste purement théorique car il semblerait, selon nos premières analyses qualitatives exploratoires, que la notion de risque soit également fonction de l'état d'avancement de la pathologie du touriste-patient.

Jacoby et Kaplan (1972) mettent en lumière six variables permettant d'identifier le risque perçu par le consommateur :

- Le risque fonctionnel : caractérisé par la différence entre les attentes à l'égard des services que le patient recevra et le prix qu'il paie en échange des services reçus.

- Le risque financier : marqué par le coût supplémentaire que le client doit assumer en cas de la défaillance du service reçu (nouvelles opérations, soins postopératoires plus longs que prévus).

- Le risque physique : pouvant se traduire par l'impact que peut engendrer le service reçu sur la santé du consommateur (réactions ou allergies à certains médicaments, incompatibilité lors d'une greffe...).

- Le risque de temps perdu : déterminé par un client qui se plaint avoir perdu son temps lors d'un séjour pouvant être considéré comme un échec pour le prestataire de service.

- Le risque social : qui est présent lorsque le service ou le produit ne correspond pas à la personnalité du consommateur (perception différentes des chirurgies esthétiques selon le contexte culturel du patient).
- Le risque psychologique : défini par l'insatisfaction générale suite à un mauvais achat.

Dans notre cas de figure, les variables dominantes concernent l'intégrité physique, morale et psychologique du consommateur. Ce sont sur ces dimensions que porte notre réflexion et à partir desquelles nous apporterons des éléments de réponse.

Plus l'incertitude et l'appréhension sont importantes et plus le risque ex ante est important (Menvielle et Menvielle, 2007). En conséquence, divers outils marketing (porte-parole, offres promotionnelles, information détaillée mais surtout témoignages des consommateurs et résultats de succès des opérations) doivent être utilisés pour induire une réaction favorable auprès du chaland et réduire ainsi le fossé existant entre sa perception des soins médicaux et la réalité de ces mêmes soins. De son côté, le consommateur cherche une information abondante pour pallier à ce risque. Ceci est d'autant plus vrai que la grande majorité des voyages à forfait touristiques de ce type sont commercialisés sur Internet et, par conséquent, un cyberconsommateur se trouve dans une démarche proactive de recherche d'information. Cette réalité est d'autant plus importante que le tourisme médical se développe dans des pays étrangers, parfois en voie de développement et ayant une connotation différente de celle associée au monde médical : Inde, Thaïlande, Singapour, pays d'Europe de l'Est, Maghreb, Afrique du Sud.

\section{Les conditions favorables à l'implantation du tourisme médical}

Plusieurs variables contribuent à la création de conditions favorables à l'implantation du tourisme médical. Nous savons que le tourisme médical coïncide avec l'apparition du tourisme durable par exemple. Cependant, les variables contribuant au développement du tourisme médical sont différentes. Leur présentation nous permettra d'élaborer un premier modèle afin de mieux comprendre les facteurs influençant le comportement du consommateur.

Au départ, le vieillissement de la population des pays développés et le phénomène du "papy boom» constituent un des vecteurs importants du développement du tourisme médical. D'ici 2015, la santé d'une grande partie des baby-boomers connaîtra un déclin progressif. Avec plus de 220 millions de baby-boomers au États-Unis, au Canada, en Europe, en Australie et en Nouvelle-Zélande, le potentiel du tourisme médical est important, rapportent Hutchison (2005) et Bennett et al. (2004). Cette analyse démographique basée sur des projections laisse entrevoir un marché abondant en termes de besoins en soins médicaux et de convalescence (Connell, 2006). En parallèle, on note aussi un intérêt des consommateurs vers des soins de nature esthétique.

Dans un deuxième temps, il faut aussi considérer l'aspect économique, l'autre variable d'importance expliquant la croissance du tourisme médical. Les prix attractifs des prestations médicales ainsi que les taux de change favorables poussent davantage les consommateurs à opter pour des soins à l'étranger (Connell, 2006). L'une des raisons essentielles de ce choix se situe dans la variable prix, constituant un argument important quand vient le temps, pour le touriste-patient, de 
Tableau 1: Options chirurgicales lourdes dans les principaux pays du tourisme médical

\begin{tabular}{|c|c|c|c|c|c|}
\hline $\begin{array}{c}\text { Types } \\
\text { d'interventions }\end{array}$ & $\begin{array}{l}\text { Montant de la prise } \\
\text { en charge par les } \\
\text { assureurs américains }\end{array}$ & $\begin{array}{l}\text { Prix de détail } \\
\text { des opérations }\end{array}$ & Inde & Thaillande & Singapour \\
\hline Pontage coronarien & 54741 à $79070^{*}$ & 122424 à 176835 & 10000 & 1200 & 20000 \\
\hline $\begin{array}{l}\text { Remplacement valvulaire } \\
\text { cardiaque (simple) }\end{array}$ & 71401 à 103136 & 159326 à 230138 & 9500 & 10500 & 13000 \\
\hline $\begin{array}{l}\text { Reconstruction } \\
\text { du genou }\end{array}$ & 17627 à 25462 & 40640 à 58702 & 8500 & 10000 & 13000 \\
\hline \multicolumn{6}{|c|}{ "Les prix indiqués sont en dollars américains. } \\
\hline
\end{tabular}

Tableau 2: Exemples de diverses prestations chirurgicales dans les principaux pays du tourisme médical

\begin{tabular}{|c|c|c|c|c|c|c|c|}
\hline Interventions* & États-Unis & Europe & Argentine & Brésil & Cuba & Inde & Bolivie \\
\hline Rhinoplastie ${ }^{\star \star}$ & 6000 & 5500 & 2300 & 2100 & 1950 & 1700 & 1200 \\
\hline Lifting du visage & 15000 & 12500 & 4300 & 4500 & 3000 & 4500 & 2600 \\
\hline Augmentation mammaire & 8000 & 7500 & 3700 & 3800 & 1600 & 3900 & 2500 \\
\hline Réduction mammaire & 9000 & 8000 & 3900 & 3600 & 2120 & 370 & 2400 \\
\hline Liposuccsion complète & 13500 & 11000 & 4500 & 4700 & 2600 & 4800 & 2700 \\
\hline Augmentation fessière & 9000 & 9000 & 4000 & 4200 & 3200 & 4500 & 3000 \\
\hline \multicolumn{8}{|c|}{$\begin{array}{l}\text { * Les prix indiqués sont en dollars américains. L’Europe comprend les pays suivants : France, Espagne et Suisse. Pour la Bolivie, il faut noter que les prix sont similaires } \\
\text { à ceux du Mexique ou du Costa-Rica. } \\
\text { *^ Chirurgie du nez pour des raisons médicales ou esthétiques. }\end{array}$} \\
\hline \multicolumn{8}{|l|}{ Source : tiré de Bovier (2008). } \\
\hline
\end{tabular}

faire des choix. Pourquoi payer cher pour un service médical alors que les patients peuvent s'offrir pour une fraction du prix seulement, le même type de soin, avec une pluralité de services associés, et bien souvent dans un cadre enchanteur? Walker (2006) souligne d'ailleurs des différences significatives en termes de chirurgies. L'Inde, par exemple, adopte un positionnement prix en matière de soins médicaux $80 \%$ moins cher que les mêmes soins dispensés aux États-Unis; pour la Thailande, ce sont des prix inférieurs de 70 à $75 \%$ qui sont offerts. Lorsqu'on sait que le système d'assurance maladie américain ne couvre qu'une fraction du coût des soins, le patient dispose d'arguments pour faire des choix éclairés. Les tableaux 1 et 2 indiquent les coûts moyens pour diverses interventions pour un patient dans différents pays du monde, en occident et dans les pays proposant des offres chirurgicales ou esthétiques diverses.

De plus, si l'on regarde les pratiques en matière de remboursement des compagnies d'assurances, il faut s'interroger sur deux aspects. Premièrement, les compagnies d'assurances américaines et anglaises ne remboursent qu'une partie des frais occasionnés par des soins. En second lieu, certains soins ne sont pas couverts par les régimes d'assurances maladie. Connell (2006) rapporte à cet effet les exemples de la chirurgie dentaire esthétique, qui n'est remboursée ni au Royaume-Uni ni en Australie.

Il faut aussi prendre en considération la longueur des listes d'attente pour une intervention chirurgicale en Amérique du Nord et au Royaume-Uni. Hutchinson (2005) a souligné cet aspect en comparant les délais pour une intervention d'une hanche. Au Royaume-Uni ou au Canada, ce délai est de l'ordre d'une année, voire parfois plus (Bovier, 2008). Le même auteur soulignait qu'en 2005, plus de 750000 Canadiens étaient sur les listes d'attentes... Les délais associés au nombre de patients poussent certains d'entre eux à se tourner vers l'Asie. Ainsi, à Bangkok (en Thaillande) ou à Bangalore (en Inde), les patients ont de grandes chances de se retrouver en salle d'opération le lendemain de leur arrivée.

Les dimensions géopolitiques constituent une des barrières les plus importantes entravant le tourisme médical. Pizam et Mansfeld (1996) signalent qu'un climat de paix 
ILLUSTRATION 3 :

Pression et niveau de contrôle des variables externes pesant sur le touriste-patient (source : données compilées par les auteurs).

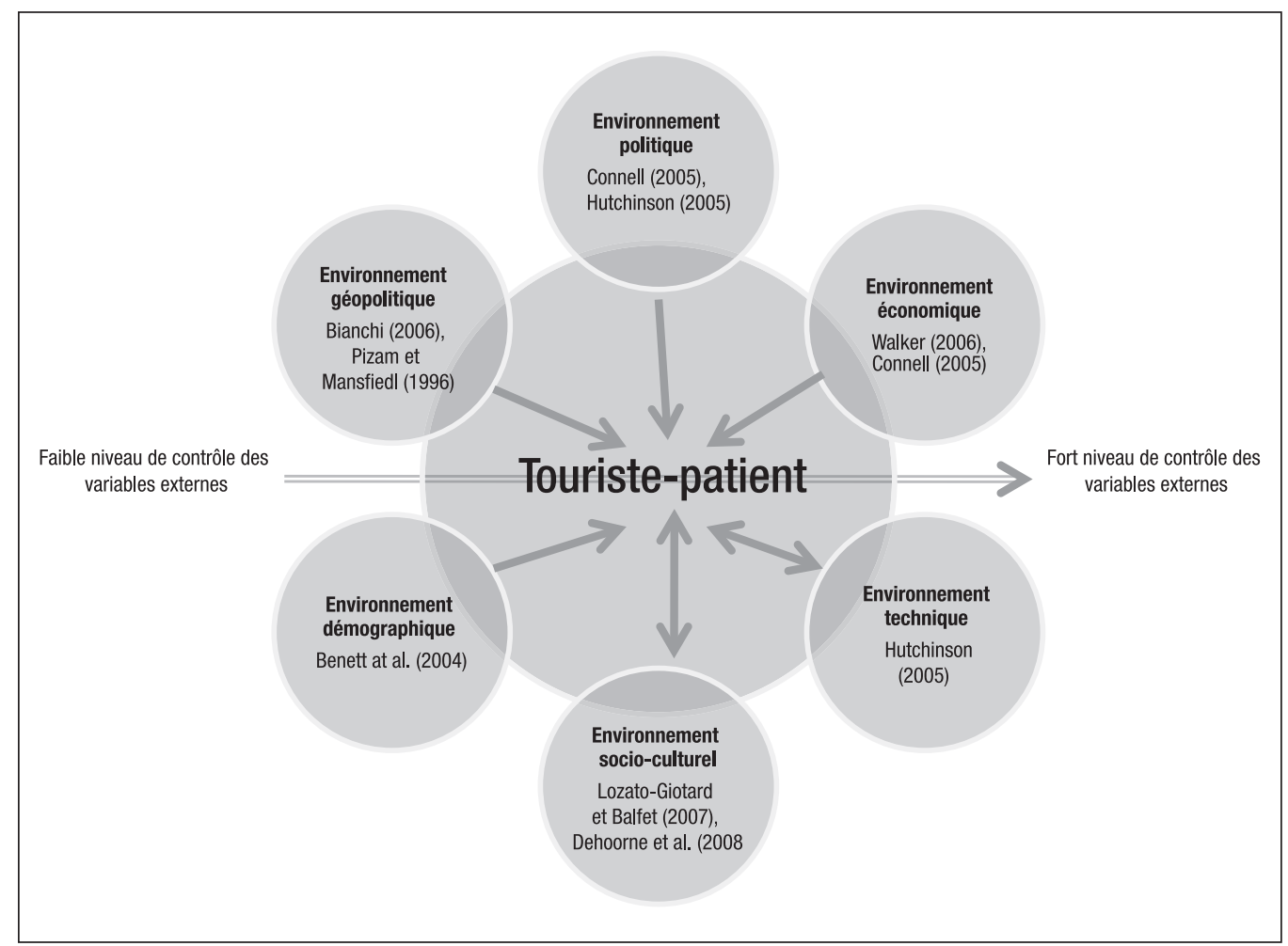

et de sécurité est un prérequis nécessaire pour attirer des touristes. Cependant cette relation de cause à effet est un peu simpliste, comme le précise Toolis (2004), la montée en puissance de groupes terroristes a contribué à altérer ce climat de confiance.

Les attaques du 11 septembre 2001 ont concouru à amplifier ce phénomène d'insécurité, d'autant plus qu'une majorité de pays s'étant ouvert au tourisme médical se trouve dans des zones où les terroristes sont potentiellement actifs. Pour Bianchi (2006), les récentes attaques par des soi-disant organisations islamiques en Indonésie, en Afrique du Nord ou en Jordanie montrent que les agressions se font souvent sans discernement, ce qui augmente encore davantage les craintes des touristes. Pforr (2006) citant Floyed et al. (2004) conclut que l'augmentation du risque perçu à l'égard d'une destination touristique est proportionnelle à l'attrait que cette dernière exerce.

La variable politique a, elle aussi, un impact sur l'offre et l'attractivité du tourisme médical. Le phénomène de déréglementation, en particulier dans le domaine du transport, a un rôle catalyseur. De la même façon, certains pays se sont désengagés de leur secteur médical, créant ainsi un nouveau modèle d'affaires. Aux États-Unis, par exemple, le secteur médical est passé des mains du public à celles du privé, engendrant ainsi des coûts importants pour les patients (Connell, 2006), notamment pour ceux n'ayant pas de protection sociale (Levasseur, 2008). On a ainsi vu l'émergence d'un système à deux vitesses davantage favorable aux citoyens capables de se payer des chirurgies loin des États-Unis, où les frais sont bien moins onéreux.
Il faut aussi prendre en compte les variables techniques ou technologiques, qui jouent un rôle de premier ordre dans le domaine du tourisme médical. Le transport aérien a démocratisé les voyages pour de nombreux touristes, qui plus est en permettant un accès à des destinations sans cesse plus lointaines. Le progrès technique a aussi permis d'améliorer la qualité du matériel médical et des soins postopératoires, offrant aux patients un plus vaste choix de destinations, parmi lesquelles ils peuvent trouver celle de prédilection. La formation des médecins et des chirurgiens entre également en ligne de compte. À titre d'exemple, l'Inde compte d'éminents chirurgiens, formés en Europe, principalement au Royaume-Uni; la Tunisie compte 8500 médecins dont les diplômes sont reconnus en Europe (Saget, 2005).

Il existe toutefois des à priori négatifs sur certains aspects. Pourtant, le niveau d'hygiène que l'on trouve dans certains pays comme la Tunisie ou l'Afrique du Sud est comparable à celui des pays occidentaux. Pour Connell (2006), les soins médicaux prodigués dans les pays pauvres pour les touristes sont comparables à ceux dispensés dans les pays occidentaux. La sécurité est la même et le personnel médical sait comment réagir face à la douleur d'un patient (Connell, 2006).

Hutchinson (2005) estime que le niveau de technologie respecte les standards médicaux et que le degré d'hygiène est très strict. À titre d'exemple, le taux de décès des patients lors d'une opération à cœur ouvert est de moins de $0,8 \%$ en Inde; il avoisine le double aux États-Unis. Il convient toutefois de mettre un bémol à ces chiffres. Dans le cadre du tourisme médical, les chirurgiens sont plus enclins à opérer des patients dont les risques de décès ou de complication sont 
faibles; ce n'est pas le cas lors d'opérations indispensables à la survie d'un patient dans les pays occidentaux.

Sur le plan socio-culturel, Dehoorne, Saffache et Tatar (2008) expliquent que les Nord-américains privilégient les offres colombiennes et costariciennes en matière de tourisme médical, alors que les Européens préfèrent des destinations méditerranéennes (Tunisie) ou asiatiques (Thaïlande, Singapour et Chine). Ces choix résultent de «liens privilégiés entre certains pays», que ces liens soient «d'ordre historique, culturel, politique, linguistique qui facilitent les relations entre certaines ex-colonies et des ex-puissances coloniales, aujourd'hui émettrices de touristes comme dans le cas des pays d'Afrique du Nord et de la France. Ce sont également les liens privilégiés entre certaines îles des Caraïbes et la France, la Grande-Bretagne ou les Pays-Bas» (Dehoorne, Saffache et Tatar : paragraphe 34 ).

L'illustration 3 dresse un bilan des propos que nous venons de formuler et de l'incidence des variables de l'environnement d'affaires sur le patient lors de son processus d'achat pour le choix d'une clinique. Dans certains cas, ces variables sont unidirectionnelles. C'est ainsi le cas des dimensions économiques ou géopolitiques, sur lesquelles les touristes n'ont que très peu, voire pas du tout d'emprise. Dans le cas des dimensions socioculturelles ou technologiques, le touriste peut avoir son mot à dire. Prenons l'exemple d'une chirurgie esthétique. Dans ce cas de figure, le patient peut «magasiner» la clinique la plus à la pointe du progrès sur le plan technologique.

Pour conclure, les pressions internes émanent de facteurs intrinsèques, liés à la motivation, la personnalité, la perception du client. D'autres facteurs extrinsèques entrent aussi en ligne de compte, comme l'influence des autres (amis, famille) c'est-à-dire du cercle d'entourage primaire du touristepatient, mais aussi de la culture (l'importance de l'apparence physique en Amérique du Nord) et les aspects de distances culturelles qui vont déterminer le choix des zones d'opérations des individus. Enfin, la notion de bouche à oreille et son pendant, les rumeurs, entretiennent aussi le processus décisionnel pour le touriste.

\section{Une stratégie de niche ou une stratégie de masse?}

À l'heure actuelle, le tourisme médical constitue une niche encore peu exploitée, mais avec un vaste potentiel de développement (Pritchard, 2003). D'autres auteurs ont abondé dans le même sens : Bennett et al. (2004) qualifient le tourisme médical de tourisme de niche disposant d'un marché aux perspectives particulièrement florissantes. Le potentiel de cette industrie est certes important, mais il doit encore être développé car il peut être considéré comme un marché à croissance rapide (Bennett et al., 2004; Connell, 2006).

Le tourisme médical a connu une première phase de démocratisation permettant ainsi d'entrevoir un "glissement» de la demande vers des catégories sociales moins élitistes. Au départ, seuls les individus les plus aisés de la société pouvaient se permettre de payer des soins dans des pays étrangers; graduellement, la classe moyenne a pu s'offrir de tels services. Le développement des moyens de transport mais aussi la pression exercée sur le touriste-patient ont contribué à cet essor. Bien évidemment, le marketing a permis de changer l'image du tourisme médical pour l'adapter aux demandes de touristes issus des autres catégories sociales.

Connell (2006) compare l'évolution du tourisme médical en ayant recours à l'exemple de la clinique Harley Street Clinic à Londres, réservée à une élite des consommateurs à la recherche des meilleurs spécialistes en cancérologie ou en chirurgie plastique. Évidemment, la dénomination de tourisme médical n'effleurait pas l'esprit des patients qui allaient dans cette clinique pour recevoir les soins de précurseurs dans le domaine.

Récemment, on a vu apparaître les forfaits complets offrant soins et hébergements vers des destinations lointaines. La vulgarisation du phénomène du tourisme médical associée aux pressions de l'environnement (coût des opérations chirurgicales, délais d'attente...) ont stimulé l'appétit des voyagistes qui n'ont pas hésité à créer des forfaits complets et sur mesure vers diverses destinations, enrichissant leur offre actuelle, comme cet exemple de combiné «safari-chirurgie». Même si ce marché représente, selon Bennett et al. (2004), une contribution importante à l'économie de nombreux pays, il faut toutefois envisager les limites et dérives de ce genre d'activité.

Dans les pays du Maghreb, la chirurgie esthétique constitue la colonne vertébrale de l'offre de ce tourisme médical, pour attirer une clientèle européenne, aisée, jeune et... féminine. En Inde, et compte tenu des opérations plus risquées proposées, c'est davantage le professionnalisme et l'expertise des chirurgiens qui sont mis de l'avant. Ces variables visent avant tout à minimiser le risque perçu chez le consommateur.

Ces deux exemples montrent à eux seuls que les stratégies marketing déployées par ces groupes de pays, proposant des services différents, toutefois regroupés sous la dénomination de "tourisme médical», visent dans les deux cas, à susciter une réaction favorable chez le consommateur, tout en suivant des voies fort différentes.

Comme on peut le voir, Internet constitue un outil de mise en marché puissant pour ces cliniques. Cependant, à l'instar d'Esnar (2005), plusieurs questions se posent lorsqu'il est question de l'utilisation de moyens de communication comme l'Internet sur le plan éthique et déontologique.

- Comment optimiser les ventes en ligne?

- Quelles sont les variables ayant une incidence positive sur le comportement d'achat?

- Comment est-il possible de réduire le risque perçu par le consommateur?

- Où est la frontière en matière d'éthique et de responsabilité sociale?

- Quel est le suivi et vers quel médecin se tourner en cas de complication?

- Quel genre de service après-vente est prévu en cas de problème de santé, suite à une vente en ligne?

\section{L'Asie, leader mondial du tourisme médical?}

Nous avons mentionné que certains pays sur l'échiquier mondial, se distinguaient des autres en proposant une offre spécialisée en matière de chirurgie ou de soins. Bien entendu, cette stratégie varie énormément d'un pays à un autre et la variable distribution engendre chez le touriste-patient une démarche attitudinale de deux types : adhésion ou aversion aux offres proposées. 

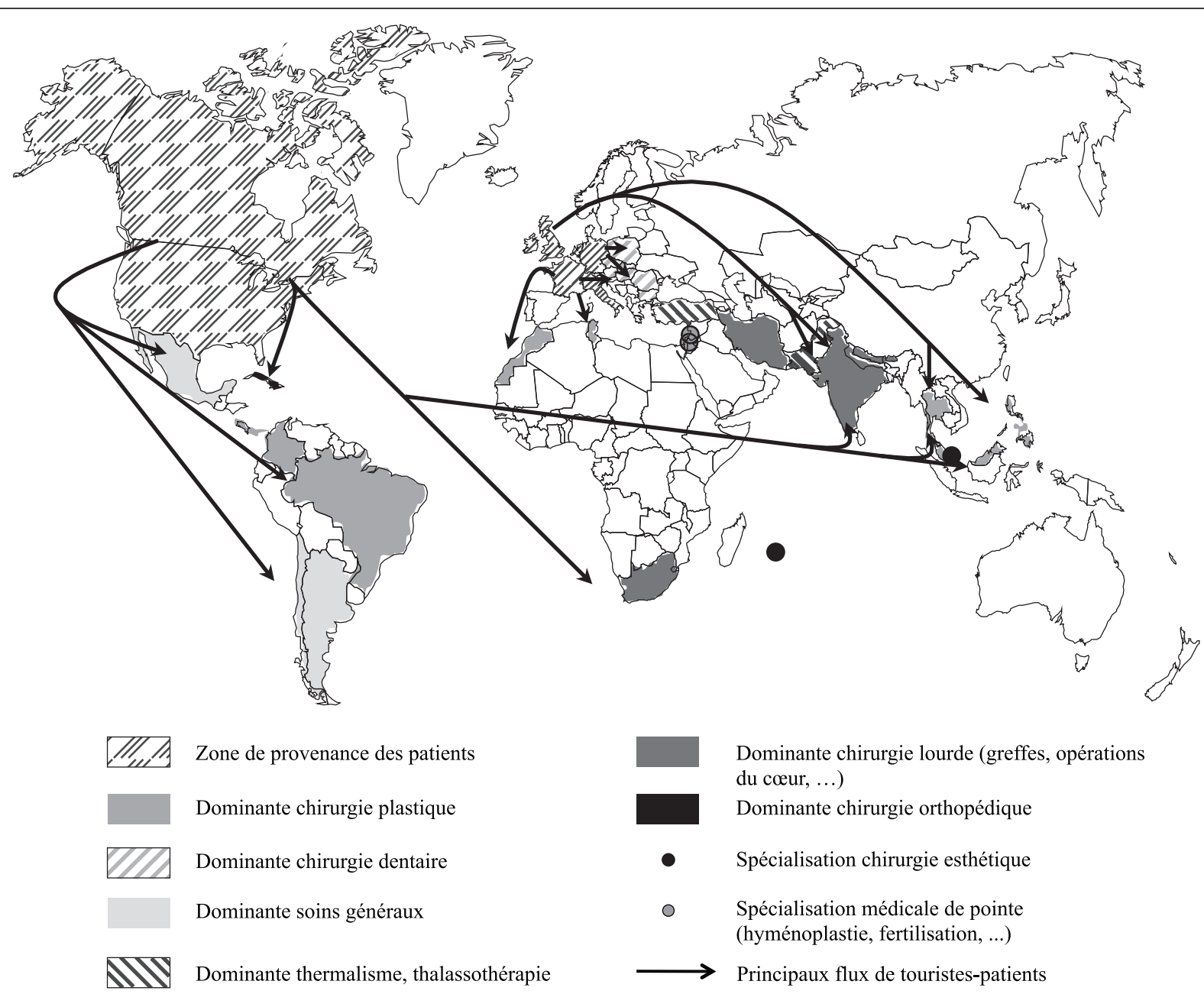

Zone de provenance des patients

Dominante chirurgie plastique

Dominante chirurgie dentaire

Dominante soins généraux

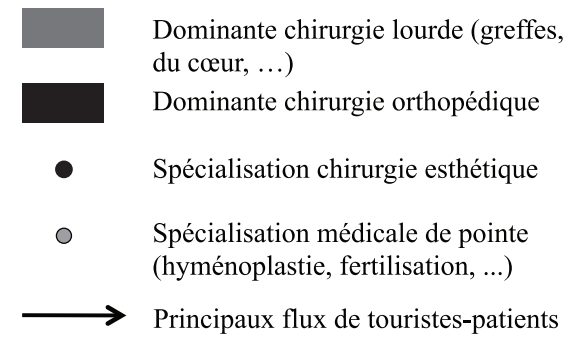

Dominante thermalisme, thalassothérapie

Principaux flux de touristes-patients

ILLUSTRATION 4 : Les courants de départ et de destination du tourisme médical (source : adapté de Courrier International (2008) et Connell (2005)).

Le Maghreb et l'Afrique du Sud se sont ainsi spécialisés dans la chirurgie plastique et esthétique. Si le nord de l'Afrique attire une clientèle francophone en provenance d'Europe, les anglophones sont davantage intéressés pas le sud de ce continent. Certains pays de l'ancien bloc soviétique comme la Pologne, la Hongrie ou la Roumanie ont développé une expertise dans la chirurgie dentaire ou oculaire attirant des patients d'Europe de l'Ouest, dont une forte majorité d'Allemands, qui $\mathrm{y}$ viennent en voisins.

Il semblerait intéressant de poser un regard sur quelques autres pays du monde, tels ceux des Caraïbes ou tous les pays bordés par l'océan, en raison du développement de la thalassothérapie, voire encore de Dubaï qui s'ouvre aux touristes fortunés et attire ceux en bonne santé, un segment de clientèle fort profitable et nécessaire à son essor économique. Au deuxième rang, le continent asiatique avec l'Inde pointe et se montre comme un leader incontesté à l'égard de cette forme de tourisme.

Les experts, tout comme les chercheurs sont unanimes pour dire que l'Inde constituera dans les années à venir, un leader dans le secteur du tourisme médical. Ces propos sont confirmés par le cabinet McKinsey et la confédération indienne de l'industrie (2006) assurant que ce pays sera un leader incontesté aussi bien en termes de chirurgie générale que de chirurgie plastique. Les projections font d'ailleurs état de revenus se situant aux alentours de 2,3 milliards de dollars d'ici 2012. Les raisons déjà évoquées dans cet article (prix attirants, capacité de logement, établissement de nouvelles cliniques, expertise des chirurgiens) concourent à valider ces chiffres. À l'appui de ces chiffres et des recherches antérieures (Menvielle, Menvielle et Tournois, 2009), le futur du tourisme médical passera par l'Asie du Sud-Est ou ne se fera pas, aurions-nous tendance à affirmer, nous appuyant sur des faits, des données statistiques (Pitti, 2009) ou certaines cartes géographiques comme l'illustration 4.

\section{En guise de conclusion}

Au cours de cet article, nous avons présenté les principales caractéristiques du tourisme médical et de ses diverses formes. Cette forme de tourisme n'est pas un fait isolé, mais bel et 
bien une nouvelle tendance, bien ancrée dans les mœurs des consommateurs et en pleine expansion. Il faut en effet souligner les éléments favorables à son émergence et à son développement tels que le prix élevé des opérations dans les pays développés, la longueur des listes d'attente, et le non-remboursement de certains frais médicaux. Le tourisme médical soulève un certain nombre de questions marketing comme la façon d'influencer le consommateur pour lui vendre ces «forfaits médicaux», les éléments du mix de communication à inclure dans un plan de communication pour stimuler la demande, ou encore les liens à tisser entre les cliniques privées et les établissements hôteliers.

Cet article a aussi permis de constater les travaux encore embryonnaires existant au niveau des chercheurs, et plus particulièrement des chercheurs européens, s'intéressant à ce sujet. Citons Bovier (2008), traçant sommairement «l'explosion» de ce nouveau type de tourisme, dont certains s'aventurent à avancer des chiffres. Ainsi, Lunt et Carrera (2010) parlent de $4 \%$ des Européens qui se feraient soigner dans un pays étranger, avec des extrémums variant de $3 \%$ au Royaume-Uni à $20 \%$ au Luxembourg. Cohen (2010) parlait de 150000 patients en Inde pour 2005 avec une progression de $15 \%$ par année (soit près de 300000 en 2010) et du même nombre pour Singapour, mais avec une augmentation de $25 \%$ (mettons le double pour 2010). Sestier et Naslafkih (2009) rapportent, quant à eux, des chiffres anciens (ceux de 2005), car les données plus récentes sont difficiles à obtenir. Ce serait donc 374000 touristes pour Singapour, plus de 150000 pour l'Inde, près de 15000 pour Israël, sans parler des prévisions faisant état de 6 millions d'Américains qui se feraient soigner à l'étranger au cours de 2010 (Neault, 2010). Le chiffre de 5 millions de touristes par année n'est donc pas loin.

Précisons aussi les quelques débats soulevés par cette nouvelle forme de voyage, qui inquiète et fait réagir certains praticiens (Brun, Deau et Roffé, 2009). Puisque de nombreuses cliniques disposent de sites Web, il pourrait être intéressant d'approfondir le thème du risque perçu des consommateurs lors de leur achat de forfaits de tourisme médical par Internet. En se plaçant du côté de la clinique maintenant, il pourrait être pertinent de comprendre la stratégie de communication déployée par ces acteurs pour faire connaître leur offre de service. Dans la continuité de la relation avec le client, quel est le service à la clientèle mis en place une fois que les soins ont été dispensés?

Dans un autre ordre d'idées, il faut aussi penser aux questions éthiques encadrant ces pratiques médicales. Dans ce questionnement, on est aussi en mesure de se demander jusqu'où il faut aller pour exploiter ce secteur d'activité. C'est d'ailleurs une des réflexions amenées par l'ordre national des médecins (Brun, Deau et Roffé, 2009) dénonçant les pratiques racoleuses de certains pays - d'Europe notamment - exerçant des pratiques médicales (IVG, dons d'ovocytes...) illégales dans de nombreux autres pays de l'Union européenne.

Même si l'Asie semble poindre avec une offre de destinations et de spécialités hors du commun, il n'en demeure pas moins que la concurrence est vive. Il faut se tourner vers Dubaï aujourd'hui pour voir l'alternative offerte aux clients des classes sociales moyennes et élevées. Comme dans biens d'autres secteurs d'activité, la concurrence est intense au niveau local (plusieurs cliniques dans un même pays), mais aussi entre les pays offrant aux patients une alternative des destinations pour signer un même problème. Dans cette dynamique, le risque de se trouver avec une médecine à deux vitesses est plus grand que jamais. Quoi qu'il en soit, pour citer Levasseur (2008), «le tourisme médical est en bonne santé».

Note

1 Citons à cet effet : http://www.lespalmiers.maghrebmed.com.tn/default. htm; http://www.lesfatimides.maghrebmed.com.tn/; http://www. cl-hammamet.maghrebmed.com.tn/ ou bien encore http://www.cl-lac. maghrebmed.com.tn/.

\section{Bibliographie}

Anonyme (2008) «Le tourisme médical est en expansion », Le Monde, $<$ http://www.lemonde.fr/planete/infographie/2008/11/25/le-tourisme-medical-est-en-expansion_1123055_3244.html>, consulté le 6 avril 2010.

BAUER, Raymond A. (1960) «Consumer behaviour as risk taking», DANS Dynamic marketing for a changing world, R.S. HANCOCK (ed.), p. 389398. Proceedings of the $43^{\text {rd }}$ Conference of the American Marketing Association,

BENNETT, Michelle; KING, Brain et Laura MILNER (2004) «The health resort sector in Australia : a positioning study", Journal of Vacation Marketing, vol. 10, n 2, p. 122-137.

BIANCHI Raoul, (2006) «Tourism and the globalisation of fear: analysis the politics of risk and (in)security in global travel», Tourism and Hospitality Research, vol 7, $\mathrm{n}^{\circ}$ 1, p. 64-74.

BOVIER Patrick A. (2008) «Explosion du tourisme médical : des voyageurs d'un nouveau type ?", Revue médicale suisse, nº 157, <http://revue.medhyg.ch/article.php3?sid=33183>, consulté le 2 avril 2009.

BOYER, Marc (1996) L'invention du tourisme, Paris : Gallimard. 336 p.

BRUN, Nicolas; DEAU, Xavier et Jean-Luc ROFFÉ (2009) «Tourisme médical : la médecine low cost?» Médecins, <http://bulletin.conseil-national. medecin.fr/article.php3?id_article=273>, consulté le 2 avril 2010.

COHEN, I. Glen (2010) Protecting Patients with Passports: Medical Tourism and the Patient Protective-Argument, Harvard Law School, Public Law \& Legal Theory, Working Paper Series, $\mathrm{n}^{\circ}$ 10-08. $76 \mathrm{p}$.

CONNELL, John (2006) «Medical tourism : Sea, sun sand and ... surgery», Tourism Management, vol. 27, $\mathrm{n}^{\circ}$ 6, p. 1093-1100.

DEHOORNE, Olivier; SAFFACHE, Pascal et Corina TATAR (2008) «Le tourisme international dans le monde : logiques des flux et confins de la touristicité", Le tourisme dans les îles et littoraux tropicaux et subtropicaux, études caribéennes, 9/10, <http://etudescaribeennes.revues.org/ document882.html\#texte>, consulté le 3 mars 2009.

ESNARD, Laure (2005) «Le boom de la chirurgie à prix réduit», Téoros, vol. 24, n 3 , automne, p. 25-29.

FLIPO, Jean-Paul (1984) Le management des entreprises de services, Paris : Les Éditions d'Organisation. 252 p.

FLIPO, Jean-Paul (1988) «On the intangibility of services», The Service Industries Journal, vol. 8, ${ }^{\circ}$ 3, p. 286-298.

FLOYED, Myron F.; GIBSON, Heather; PENNINGTON-GRAY, Lori et Brijesh THAPA (2004) «The effect of risk perceptions on intentions to travel in the aftermath of September 11,2001", Journal of Travel and Tourism Marketing, vol. 15, n 2/3, p. 19-38.

GARCIA-ALTES, Anna (2005) «The development of health tourism services», Annals of Tourism Research, vol. 32, n 1, p. 262-266. 
GARTNER, William, C. et David W. LIME (2000) Trends in Outdoor Recreation, Leisure and Tourism, Cambridge : CABI Publishing. 496 p.

GILBERT, Edmund W. (1954) Brighton: Old ocean's bauble, London : Methuen \& Co. Ltd. 275 p.

GÜSEMAN, Dennis S. (1981) «Risk perception and risk reduction strategies in consumer services", DANS Marketing of services, DONELLY J.A. et W.R. GEORGE (éd.), p. 200-204. Chicago : American Marketing Association.

HUTCHINSON, Becca (2005) «Medical tourism growing worldwide», Messenger - University of Delaware Review, vol. 13, $\mathrm{n}^{\circ} 4,<\mathrm{http} / / / \mathrm{www}$. udel.edu/PR/UDaily/2005/mar/tourism072505.html>, consulté le 24 juillet 2007.

JACOBY, Jacob et Leon B. KAPLAN (1972) «The Components of Perceived Risk", DANS Proceedings of the 3rd Annual Conference, Venkatesan M. (éd.), p. 382-93. Chicago : Association for Consumer Research, College Park, MD.

KALFON, Pierre (2009) Tourisme et innovation, Paris : L'Harmattan. 130 p.

LAROCHE, Michel; BERGERON Jasmin et Chistine GOUTALAND (2001) "A three-dimensional scale of the intangibility", Journal of Service Research, vol. 4, n 1, p. 26-38.

LAROCHE, Michel; BERGERON, Jasmin et Chistine GOUTALAND (2003) «How intangibility affects perceived risk: the moderating role of knowledge and involvement », Journal of Services Marketing, vol. 17, $\mathrm{n}^{\circ} 2$, p. 122-140.

LAUTIER, Marc (2007) « Les pays en développement et le commerce international de services de santé : Une comparaison Tunisie-Jordanie ", Économies et sociétés, série EGS, n 8 , 3/2007, p. 489-519. Repris dans Problèmes économiques, $\mathrm{n}^{\circ} 214$, septembre 2007.

LE BORGNE, Catherine (2007) «Le tourisme médical : une nouvelle façon de se soigner ", Sève : Presses de Sciences Po, vol. 2, nº 15, p. 47-53, <http://www.cairn.info/article.php?ID_REVUE=SEVE\&ID_ NUMPUBLIE=SEVE_015\&ID_ARTICLE=SEVE_015_0047>, consulté le 2 avril 2010.

LEVASSEUR, Maïthé (2008) «Tourisme médical: opportunités et controverses", Réseau de veille en tourisme, 12 septembre, <http:// veilletourisme.ca/2008/09/12/tourisme-medical-opportunites-etcontroverses/?tagged $>$, consulté le 2 octobre 2008.

LOZATO-GIOTARD, Jean-Pierre et Michel BALFET (2007) Management $d u$ Tourisme, $2^{\mathrm{e}}$ édition, Paris : Pearson Education. 384 p.

LUNT, Neil et Percivil CARRERA (2010) «Medical tourism: Assessing the evidence on treatment abroad», Maturitas, sous presse.

MALLET, Stéphane (2004) À la recherche des origines du risque perçu à l'égard de l'achat d'un produit: Colloque Société et Consommation, $3^{e}$ Journée normande de la consommation (11 et 12 mars 2004).

MCKINSEY et The Hindu Business Line (2006) «Confederation of Indian Industry initiative to promote health tourism in Kerala», $<$ http://www. thehindubusinessline.com/2006/03/07/stories/2006030702271900.htm>, consulté le 23 juillet 2007.

MENVIELLE, Loick et William MENVIELLE (2007) «Medical Tourism: a new way of traveling», 3rd International Conference on Tourism (juillet), Athènes, Grèce : ATINER.
MENVIELLE, Loick; MENVIELLE, William et Nadine TOURNOIS (2009) "Comment diminuer le risque perçu chez les cyberconsommateurs, lors de l'achat de services en ligne : une étude exploratoire des sites Web dédiés au tourisme médical», Actes de la $2^{e}$ Journée de Recherche sur le Tourisme, La Rochelle (France).

MENVIELLE, Loick; MENVIELLE, William et Nadine TOURNOIS (2010) "Pôles de compétitivité et régionalisation de la santé », DANS Pôles de compétitivité : piloter et performer des réseaux d'innovation, B. ALIOUAT, Paris : Hermes Science Publishing, (à paraître).

MUELLER, Hansrüdi et Evelyn LANZ KAUFMANN (2001) «Wellness tourism : Market analysis of a special health tourism segment and implications for the hotel industry», Journal of Vacation Marketing, vol. 7, $\mathrm{n}^{\circ} 1$, p. 5-17.

MURRAY, Keith B. et John L. CHLATER (1990) «The impact of services versus good on consumers' assessment of perceived risk and variability», Academy of Marketing Science, vol. 18, n 1, p. 51-66.

NEAULT, Chantal (2010) «Si le tourisme médical vous intéresse : quelques stratégies d'adaptation de l'hébergement", Réseau de veille en tourisme, <http://veilletourisme.ca/2010/04/09/si-le-tourisme-medical-vous-interesse-quelques-strategies-d\%e2\%80\%99adaptation-de1\%e2\%80\%99hebergement/>, consulté le 11 avril 2010.

Organisation mondiale du tourisme (2010) Baromètre OMT du tourisme mondial, vol. 8, $\mathrm{n}^{\circ}$ 1, janvier 2010, <http://www.unwto.org/facts/eng/ barometer.htm>, consulté le 3 avril 2010.

PFORR, Christof (2006) «Tourism in Post-Crisis is tourism in Pre-Crisis : a review of the Literature on Crisis Management in Tourism ", Curtin University of Technology - School of Management, Working Paper Series, $n^{\circ}$ 2006-1.

PITTI, François (2009) «Chine et Inde : vers une stratégie de marque», En Temps Réel - Cahier n ${ }^{\circ} 38$, avril, <http://www.entempsreel.com/ sites/default/files/En\%20Temps\%20Réel\%20-\%20Cahier\%2038.pdf>, consulté le 10 avril 2010.

PIZAM, Abraham et Yoel MANSFELD (1996) Tourism, Crime and International Security Issues, Chichester : John Wiley \& Sons. 330 p.

PRITCHARD, Christian (2003) «Asian nations fight for tourists' health dollars», Medical Post, Toronto, 18 novembre, vol. 39, n 42, p. 54.

PROULX, Luce (2005) «Tourisme, santé et bien être», Téoros, vol. 24, no 3 , p. 5-11.

SAGET, Estelle (2005) «Des pays très hospitaliers», L'Express, octobre, $<$ www.lexpress.fr/reussir/dossier/sante2/dossier.asp?ida=434541>, consulté le 8 octobre 2008.

SESTIER, François et Abdelouahed NASLAFKIH (2009) «Le tourisme médical sous le microscope", Forum 2009 de l'Institut Canadien de la retraite et des avantages sociaux (ICRA), 15 septembre.

SMITH, Melanie et Catherine KELLY (2006) «Wellness Tourism», Tourism Recreation Research, vol. 31, $\mathrm{n}^{\circ}$ 1, p. 1-4.

TOOLIS, Kevin (2004) «Rise of the terrorist professors», New Statesman, 14 juin, p. 26-27.

WALKER, Tracy (2006) «Consumers go abroad in pursuit of cost-effective healthcare», Managed Healthcare Executive, juillet, vol. 16, nº 7, p. 10. 